[0212-7199 (2007) 24: 5; pp 235-238] ANALES DE MEDICINA INTERNA Copyright (C) 2007 ARAN EDICIONES, S.L.

AN. MED. INTERNA (Madrid) Vol. 24, N. ${ }^{\circ}$ 5, pp. 235-238, 2007

\title{
Insuficiencia renal aguda por depósito de cristales de Sulfadiacina
}

\author{
F. J. DE LA PRADA ÁLVAREZ, A. M. PRADOS GALLARDO ${ }^{1}$, A. TUGORES \\ VÁZQUEZ, M. URIOL RIVERA, A. MOREY MOLINA \\ Servici de Nefrología. Hospital Universitario Son Dureta. Palma de Mallorca. ${ }^{1}$ Servicio \\ de Medicina Interna. Hospital Universitario Virgen Macarena. Sevilla
}

ACUTE RENAL FAILURE DUE TO SULFADIAZINE CRYSTALLURIA

\begin{abstract}
RESUMEN
La encefalitis necrotizante focal por Toxoplasma Gondii es una de las infecciones oportunistas más frecuente en pacientes con síndrome de inmunodeficiencia adquirida. (SIDA). El tratamiento de elección consiste en la combinación de Pirimetamida y Sulfadiacina. Uno de los principales efectos adversos de la Sulfadiazina es su precipitación en el sistema urinario. Presentamos a una paciente con SIDA y encefalitis por Toxoplasma Gondii que desarrolla insuficiencia renal aguda reversible por depósito de cristales de sulfadiacina a nivel renal. La ecografía mostró múltiples calcificaciones en el seno renal, que desaparecieron tras hidratación y administración de bicarbonato sódico endovenoso. Estos pacientes y otros inmunodeprimidos, presentan factores favorecedores de la precipitación de éste y otros fármacos, siendo necesario una adecuada monitorización de la función renal y del sedimento urinario, junto con medidas profilácticas como el aumento de la ingesta de líquidos y la alcalinización urinaria.
\end{abstract}

PALABRAS CLAVE: Sulfadiacina. Cristaluria. Insuficiencia renal aguda.

\begin{abstract}
Focal necrotizing encephalitis due to Toxoplasma gondii infection represents one of the most common oportunistic infection in patients with the acquired inmunodeficiency syndrome (AIDS), and the treatment is commonly with a combination sulphadiazine, and pyrimethamine. A major side effect of sulfadiazine therapy is the occurrence of cristallization in the urinary collecting system. We report a patient with AIDS and Toxoplasmic encephalitis treated with sulfadiazine who developed acute renal failure. Renal ultrasound demonstrated echogenic areas within the renal parenchyma, presumed to be sulfa crystals. Renal failure and ultrasound findings resolved rapidly with hidratation and administration of alkali. Patients infected with AIDS frequently have characteristic that increase intratubular crystal precipitation and they require treatment with one or more of the drugs that are associated with crystal-induced renal failure. Controlled alkalinization of the urine and high fluid intake are recommended for prophylaxis of crystalluria. The literature concerning crystalluria and renal failure due to sulfadiazine is reviewed.
\end{abstract}

KEY WORDS: Sulphadiazine. Crystalluria. Renal failure.

De la Prada Álvarez FJ, Prados Gallardo AM, Tugores Vázquez A, Uriol Rivera M, Morey Molina A. Insuficiencia renal aguda por depósito de cristales de Sulfadiacina. An Med Interna (Madrid) 2007; 24: 235-238.

\section{INTRODUCCIÓN}

La sulfadiacina es una sulfamida utilizada actualmente en el tratamiento de la toxoplasmosis cerebral en pacientes con SIDA. Presenta escasa solubilidad en medio ácido y en éstos enfermos pueden concurrir otras situaciones predisponentes para su precipitación en el sistema urinario.

Presentamos el caso de una paciente con toxoplasmosis cerebral en tratamiento con sulfadiacina y pirimetamida, que presenta daño tubular renal por precipitación de cristales de sulfadiacina. Describimos las características farmacocinéticas que influirán en la eliminación renal del asulfadiacina, y los factores de riesgo, el tratamiento y las medidas profilácticas disponibles para evitar dicha toxicidad.

\section{CASO APORTADO}

Paciente mujer de 38 años de edad con antecedentes personales de hábito tabáquico (40 cigarrillos/día), esplenectomizada tras traumatismo hace 21 años, ex-adicta a drogas por vía parenteral desde hace 3 años, y en tratamiento actual con metadona. Diagnosticada de 
infección por VIH hace 5 años (VIH G C3), realizando tratamiento con Lamivudine $300 \mathrm{mg} /$ dia, zidovudine $600 \mathrm{mg} /$ dia y efavirenz 600 $\mathrm{mg} /$ día. Dos meses antes del ingreso en nuestro servicio inicia tratamiento con pirimetamina $50 \mathrm{mg} /$ día, sulfadiacina 4 gramos/día y ácido folico $15 \mathrm{mg} /$ día a raíz de cuadro de toxoplasmosis cerebral. La función renal previa era normal (creatinina $0,79 \mathrm{mg} / \mathrm{dl}$, urea 15 $\mathrm{mg} / \mathrm{dl}$ ) y 10 días antes de nuestro ingreso se le realizó una ecografía abdominal presentando riñones de tamaño, morfología y ecogenicidad normales.

Consulta por cuadro de 10 días de evolución de náuseas, vómitos y deposiciones diarreicas de 48 horas de evolución, negando disminución del volumen de diuresis en los días previos al ingreso.

A la exploración la paciente está afebril, presenta un regular estado general con lengua seca y signo del pliegue positivo. Su tensión arterial es de 90/60 mmHg. La auscultación cardíaca objetiva tonos rítmicos a $96 \mathrm{lpm}$; auscultación respiratoria sin ruidos patológicos; abdomen blando, depresible, no doloroso, sin masas ni visceromegalias, con peristaltismo conservado y sin soplos vasculares. Los pulsos centrales y periféricos son simétricos y no hay edemas.

En las exploraciones complementarias se detectan signos de insuficiencia renal con urea $136 \mathrm{mg} / \mathrm{dl}$ y creatinina $6.8 \mathrm{mg} / \mathrm{dl}$; acidosis metabólica con GAP elevado y alcalosis metabólica asociada, secundarias respectivamente a Insuficiencia renal y alcalosis de contracción; hipoproteinemia $(46,3 \mathrm{~g} / \mathrm{l})$ e hipoalbuminemia $(27,6 \mathrm{~g} / \mathrm{l})$, con hemograma y resto de parámetros plasmáticos normales. El sedimento de orina muestra hematuria microscópica, leucocituria sin bacteriuria, y abundantes cristales en forma de gavilla de trigo. En orina el $\mathrm{pH}$ es de 5,0 , el sodio urinario de $53 \mathrm{mEql} / 1$ y la excreccion fracional de sodio de $11,4 \%$.

Se realizó ecografía abdominal que mostró ambos riñones discretamente aumentados de tamaño, con aumento de la ecogenicidad cortical y múltiples calcificaciones en el seno renal, sin signos de uropatía obstructiva (Fig. 1).

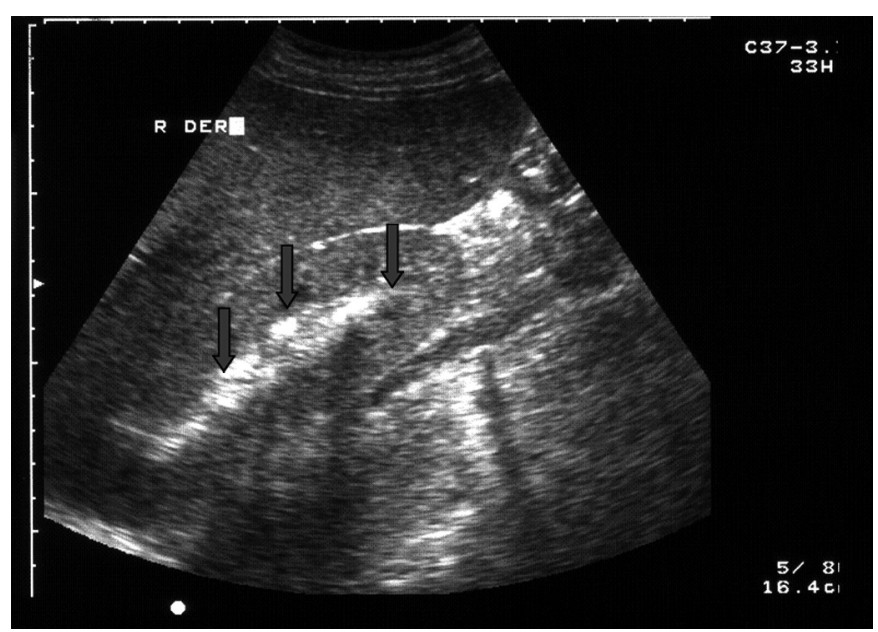

Fig. 1. Ecografía de riñón derecho con imágenes de calcificación en seno renal (flechas) y sombra sónica posterior.

Ante estos hallazgos y con el diagnóstico clínico de insuficiencia renal aguda por depósito de cristales de sulfadiacina, se inició tratamiento con sueroterapia enérgica, $3.000 \mathrm{cc} / 24$ horas de suero salino $0,9 \%$ para rehidratación y $1.000 \mathrm{cc} / 24$ horas de suero bicarbonato $1 / 6$ molar para alcalinizar la orina, con control de diuresis mediante sondaje vesical. La diuresis desde el ingreso osciló entre 140 y 160 $\mathrm{ml} /$ hora y se alcanzó un $\mathrm{pH}$ urinario igual o mayor a 7,5. Después de 9 días con sueroterapia se consigue la normalización de los valores de urea y creatinina plasmáticos. Tras la normalización de la función renal se mantuvo el tratamiento con Pirimetamina y se añadió Clindamicina $300 \mathrm{mg} / 8$ horas.

A los 15 días del ingreso se realiza ecografía abdominal de control mostrando riñones de tamaño y ecogenicidad normal, con buena diferenciación corticomedular sin dilatación del sistema excretor y sin evidencia de las calcificaciones previamente descritas (Fig. 2).

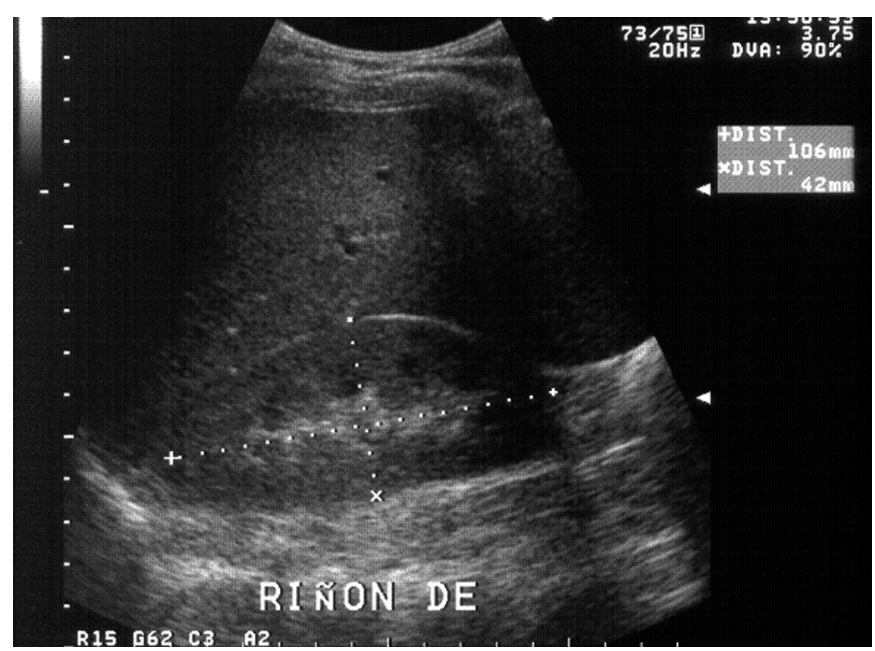

Fig. 2. Ecografía de riñón derecho sin imágenes de calcificación tras el tratamiento.

\section{DISCUSIÓN}

El fracaso renal agudo por cristales de sulfamidas fue una complicación relativamente frecuente en la década de los años 1930-40, pero su incidencia disminuyó con la aparición de sulfonamidas mas solubles y de otros antibióticos mas eficaces. Desde el final de la década de 1980 se han vuelto a comunicar nuevos casos debido a su indicación en el tratamiento de la encefalitis necrotizante focal secundaria a Toxoplasma gondii en pacientes VIH-positivos (1), y en el tratamiento de la Nocardiosis pulmonar (2).

La sulfadiacina es una sulfamida de vida media corta $(7 \pm$ 3,9 horas), cuyo mecanismo de acción es inhibir el crecimiento bacteriano al inhibir la síntesis de ácido fólico a través de un antagonismo competitivo por el PABA. Tras una rápida absorción oral, se une a la albúmina en mas de un $55 \%$, por lo que la hipoalbuminemia aumenta las concentraciones plasmáticas y urinarias del fármaco libre, aumentando la posibilidad de cristalización (3). Tiene una amplia distribución tisular, sufriendo acetilación hepática parcial y rápida eliminación urinaria en altas concentraciones, 40-60\% como fármaco y $15-40 \%$ como metabolito acetilado. Ambas formas tienen escasa solubilidad en medio ácido. Su solubilidad en orina depende de la concentración del fármaco en plasma y orina, de la cantidad de fármaco en forma acetilada, del estado de hidratación del paciente, del $\mathrm{pH}$, del volumen y temperatura orina. De entre todos estos factores la solubilidad es muy dependiente del $\mathrm{pH}$ urinario, y el nivel al que suele desarrollarse nefrotoxicidad es de 5,5, siendo el $\mathrm{pH}$ de 7,15 el valor critico de cristalización $(1,4)$. 
La solubilidad en orina de la sulfadiacina y de su principal metabolito, la acetilsulfadiacina, es casi diez veces mayor a $\mathrm{pH} 7,5(200 \mathrm{mg} / \mathrm{ml}$ y $512 \mathrm{mg} / \mathrm{dl}$ respectivamente), que a pH de 6,5 (28 mg/dl y $75 \mathrm{mg} / \mathrm{dl})$ (5). A dosis usuales de 4 gramos al día, son necesarios 16 litros de orina a $\mathrm{pH}$ 5,5 para disolver todo el fármaco. Mientras que el aumento del volumen urinario de 1 a 3 litros sólo duplicará la solubilidad de la sulfadiazina en la solución, un aumento del pH urinario de 5,5 a 7,5 aumentará la solubilidad en unas 15 a 20 veces, siendo ésta la medida más eficaz para la profilaxis y el tratamiento.

Esta complicación suele aparecer después de una media de 7-10 días de tratamiento, pero se han descrito casos desde el inicio del mismo hasta dos meses después. La dosis acumulada del fármaco en el momento de la toxicidad renal suele ser de 40 gramos aunque pueden aparecer con menos (1).

La incidencia total de toxicidad renal por sulfadiazina varía entre 1 y $32 \%$. La incidencia de fracaso renal agudo puede llegar a ser del $29 \%$, la de cristaluria varía entre 0,4 y $49 \%$, la hematuria entre 1 y $32 \%$, y los cálculos renales entre 0,4 y $20 \%$ (1).

En enfermos con SIDA esta complicación es más frecuente (1,9-7,5 vs. $1-4 \%)$, suele ser más tardía (21 vs. 10 días) y con mayor dosis acumulada (84 vs. 40 gramos) que en pacientes HIV-negativos (4).

Los enfermos con SIDA presentan muchos de los factores de riesgo para el desarrollo de esta complicación, como son el largo tiempo de tratamiento a dosis elevadas, la hipoalbuminemia, la incapacidad de mantener un buen estado de hidratación (por disminución del nivel de conciencia, anorexia, disfagia, vómitos, diarreas y/o fiebre, con disminución del volumen urinario), la alteración de la función renal previa que puede pasar inadvertida por niveles plasmáticos "normales" de creatinina en pacientes caquécticos, y el uso concomitante de otros fármacos que inducen cristaluria como aciclovir, otras sulfonamidas, metotrexate, indinavir, triamterene, piridoxilato, primidona, ciprofloxacino, vitamina $\mathrm{C}$, foscarnet, cefalexina, ampicilina, xylitol y ácido acetilsalicílico, pudiendo explicar una mayor incidencia de nefrotoxicidad por sulfadiacina en estos pacientes (6).

Los cristales de sulfadiacina pueden depositarse a cualquier nivel del tracto urinario, por lo que la clínica de presentación es variada, siendo lo más frecuente el dolor abdominal $(80 \%)$, con mayor frecuencia localizado en flancos $(51 \%)$ o a nivel lumbar $(26 \%)$, acompañándose de oliguria (46\%), anuria $(14 \%)$, hematuria macroscópica (43\%) y/o disuria (23\%). Un 3\% de los casos presentan cristaluria asintomática (4).

Estos cristales provocan abrasión mecánica local e irritación química del epitelio del tracto urinario, provocando daño celular consistente desde el punto de vista histológico en hemorragia peritubular, infiltración leucocitaria, necrosis tisular y tardíamente depósitos de calcio. Estos cristales pueden además formar cálculos a cualquier nivel del tracto urinario responsables de posibles obstrucciones (2).

El diagnóstico es clínico y ecográfico. En el sedimento de orina se suele detectar cristaluria $(91 \%)$ con su característica forma de gavillas de trigo con unión excéntrica, formados por agregados del principal metabolito, la $\mathrm{N}$-acetil-sulfadiazina, pudiendo aparecer también eritrocitos (72\%), leucocitos $(41 \%)$, proteinuria $(22 \%)$ y cilindros (3\%) (4). La aparición de cristaluria aislada no es indicativa de la presencia de daño renal, pero debe inducir a una monitorización extrema de la orina, con el objeto de detectar hematuria o piuria, que si lo indicarían (1). El test de Lignin puede ser utilizado para detectar sulfonamidas en la orina, y consiste en mezclar una gota de ácido clorhídrico al $10 \%$ con una gota de orina obteniéndose una coloración anaranjada en presencia de cristales de sulfonamidas (7)

La radiología convencional tiene poca sensibilidad diagnóstica. En la ecografía es frecuente encontrar densidades tipo cálculo $(77 \%)$ e hidronefrosis $(50 \%)$, aunque puede resultar normal (19\%) (4). El diagnóstico diferencial ecográfico incluye cualquier otra causa de cálculos calicilares, pero la afectación uniforme en todos los cálices y la desaparición con el tratamiento médico es muy sugestivo de deposito de cristales a nivel renal (8).

Una vez establecido el fallo renal debe aumentarse la diuresis con sueroterapia, alcalinizar la orina para alcanzar un $\mathrm{pH}$ $>$ 7,15 mediante bicarbonato sódico endovenoso, siendo esta la medida más eficaz, controlándose el $\mathrm{pH}$ y el bicarbonato plasmático para evitar sobrecargas en pacientes con insuficiencia renal aguda. Puede sustituirse la sulfadiazina por clindamicina, pero es posible la resolución del proceso manteniendo el mismo tratamiento antibiótico (9) con la reducción de la dosis (10) o su reintroducción tras la resolución del cuadro (1,4,10-12). Si persiste la anuria debe sospecharse obstrucción urinaria por cálculos, pudiendo ser necesaria la cateterización ureteral e irrigación de la pelvis renal con bicarbonato sódico o nefrostomías percutaneas $(3,6,13,14)$. A veces ha sido necesario el tratamiento renal sustitutivo (17). Tras el tratamiento la recuperación de la función renal suele ser rápida y completa, en un período de 2 a 21 días con una media 6 , incluso en los pacientes en los que se reduce la dosis (4).

Las medidas profilácticas deberían aplicarse a todos los pacientes que reciben estos fármacos. Antes de iniciar el tratamiento es necesario comprobar la función renal mediante el aclaramiento de creatinina y la integridad de la vía excretora. Debe evitarse la asociación de otros fármacos nefrotóxicos, prevenir la hipovolemia instruyendo a los pacientes a aumentar la ingesta de líquidos con el fin de mantener una diuresis de 3 litros/día. Hay que mantener un ph urinario alcalino ( $\mathrm{pH}$ $>7,15$ ) administrando 12 gramos de bicarbonato sódico, habiéndose considerado esta medida incluso en profilaxis primaria. Debe monitorizarse durante el tratamiento la función renal y la aparición de cristaluria y/o microhematuria, sobre todo en las primeras semanas. La monitorización de los niveles plasmáticos de sulfadiacina, para mantenerlos por debajo de $15 \mathrm{mg} / \mathrm{dl}$, no garantiza la ausencia de complicaciones renales, aunque si es aconsejable su determinación en pacientes con daño renal previo $(1,4)$.

En nuestro paciente interpretamos que la incapacidad de mantener una ingesta líquida adecuada, la hipoproteinemia y el ph urinario ácido, favorecieron la cristalización de la sulfadiacina a nivel urinario provocando daño tubular.

La nefrotoxicidad por Sulfamidas, que había sido ya olvidada, ha vuelto a reaparecer ante la epidemia que supone el Síndrome de Inmunodeficiencia Adquirida. Dado que los pacientes con riesgo de desarrollar esta complicación son tratados por otros especialistas diferentes al Nefrólogo, aquellos deberían estar formados y atentos a la posible aparición de la misma. 


\section{Bibliografía}

1. Simon DI, Brosius FC $3^{\text {rd }}$, Rothstein DM. Sulfadiazine crystalluria revisited. The treatment of Toxoplasma encephalitis in patients with acquired immunodeficiency syndrome. Arch Intern Med 1990; 150: 2379-84.

2. Portoles J, Torralbo A, Prats D, Blanco J, Barrientos A. Acute renal failure and suphadiazine crystalluria in kidney transplant. Nephrol Dial Transplant 1994; 9: 180-1.

3. Sahai J, Heimberger T, Collins K, Kaplowtiz L, Polk R. Sulfadiazine induced crystalluria in a patient with AIDS: A reminder. Am J Med 1988; 84: 791-2.

4. Becker K, Jablanowsky H, Haussinger D. Sulphadiazine associated nephrotoxicity in patients with the acquired inmunodeficiency syndrome. Medicine, Baltimore 1996; 75: 185-94.

5. Goodman GA, Rall TW, Nies AS, Taylor P. The pharmocological basis of thrapeutics, 8th edition. New York: Mc Graw Hill, 1990: 1047.

6. Perazella M. Cristal-induced Acute renal Failure. Am J Med 1999; 106: $459-65$.

7. Carbone LG, Bendixen B, Appel GB. Sulfadiazine-associated obstructive nephropathy occurring in a patient with the acquired immune deficiency syndrome. Am J Kidney Dis 1988; 12: 72-5.

8. Sasson JP, Dratch PL, Shortsleeve MJ. Renal US findings in sulfadiazine-induced crystalluria. Radiology 1992; 185: 739.
9. Marques LP, Silva MT, Madeira EPQ, Santos OR. Obstructive renal failure due to therapy with sulfadiazine in a AIDS patient. Nephron 1992; 62: 361 .

10. Oster S, Hutchison F, McCabe R. Resolution of acute renal failure in toxoplasmic encephalitis despite continuance of sulfadiazine. Rev Infect Dis 1990; 12: 618-20.

11. Carbone LG, Bendixen B, Appel GB. Sulfadiazine-associated obstructive nephropathy occurring in a patient with the acquired immune deficiency syndrome. Am J Kidney Dis 1988; 12: 72-5.

12. Zubizarreta J, Ayensa D, Aldamiz-Echevarria M, Ruiz de Gauna R. Insuficiencia renal aguda secundaria a cristaluria por sulfadiazina. Med Clin 1994; 102: 76-7.

13. Hein R; Brunkhorst R; Thon WF; Schedel I; Schmidt RE. Symptomatic sulfadiazine crystalluria in AIDS patients: A report of two cases. Clin Nephrol 1993; 5: 254-6.

14. Crespo M, Quereda C, Pascual J, Rivera M, Clemente L, Cano T. Patterns of sulfadiazine acute nephrotoxicity. Clin Nephrol 2000; 54: 6872 .

15. Christin S, Baumelou A, Bahri S, Ben Hmida M, Deray G, Jacobs C. Acute renal failure due to sulfadiazine in patients with AIDS. Nephron 1990; 55: 233-4. 